\title{
Ultrasonographic Detection of Interstitial Nephritis in a Canine Fetus
}

\author{
Priscila Silva', Marjury Cristina Maronezi', Beatriz Gasser', Letícia Pavan', Luiz Paulo Nogueira Aires', Luciana \\ Cristina Padilha-Nakaghi' ${ }^{1}$ Ricardo Andrés Ramirez Uscategui ${ }^{3}$ \& MarcusAntônio Rossi Feliciano ${ }^{1,2}$
}

\begin{abstract}
Background: A detailed ultrasound examination of the fetal urinary tract as part of prenatal care is important to detect possible abnormalities. Early diagnosis can prevent more serious damage such as loss of kidney function. Interstitial nephritis can compromise renal functionality. Also, acute interstitial nephritis is a frequent cause of acute kidney injury and can become chronic if not treated. Besides, a renal biopsy can help in the diagnosis and at the staging of the disease. Therefore, this report aims to describe the ultrasonographic findings of the renal tissues in a canine fetus affected by interstitial nephritis. Case: Gestational ultrasound evaluations were performed in a female Pug, at the 25th, 45th, and 58th gestational days. Ultrasound examination was performed with a $9.0 \mathrm{MHz}$ linear transducer and a high-resolution digital transducer with a frequency standardized at 17.0 MHz. During the first evaluation, embryonic vesicles were detected and were compatible with the gestational period. At 45 days of gestation, some abnormalities were observed in the kidneys of one of the fetuses, such as increased cortical echogenicity, cortical thickening, loss of corticomedullary ratio (1:1), renal pelvis and ureter dilatation by echogenic content. Biparietal and abdominal diameters were also lower than that from the other fetuses, which did not show abnormalities, being compatible with the estimated gestational age. However, the heart rate was similar among all fetuses. On the 58th day, we observed in the underdeveloped fetus that both kidneys still presented the abnormalities seen previously, in addition to the presence of a cystic structure in the right kidney, in the cranial topography of the cortex. The other fetuses kept showing normal ultrasonographic findings, normal heartbeat, all of these compatible with the ultrasound gestational age. The delivery occurred without complications and only the underdeveloped fetus stillborn. Then, it was submitted to a necropsy where chronic interstitial nephritis was found in the histopathological analysis.

Discussion: Adequate prenatal care is important to detect abnormalities in fetuses, in which ultrasound examination allows to access fetal development. The gestation in the bitch seemed to be occurring without complications, but the B mode and High Definition ultrasound techniques were able to identify abnormal development of the fetus' kidneys. Both techniques showed to be secure to the fetuses and the mother, as they are non-invasive and free of radiation. These findings, different from those described as normal for a fetus, reinforce the warning signal for a possible establishment of anomalies consistent with nephropathy. Still, histopathology is important to exclude other diseases and help to define prognosis. Interstitial nephritis can significantly affect perinatal survey, considering that the fetus was stillborn, confirming that the description of such anomalies can help to prevent disease. Since nephritis can be associate with the use of some medicines, the orientation of the owner regarding drug use during bitch pregnancy is essential. Therefore, this is the first report describing renal abnormalities in a canine fetus, which highlights the importance of ultrasound examination for disease identification in prenatal care. Lastly, high-resolution ultrasound (HD) could provide detailed information on abnormalities in the fetal kidney.
\end{abstract}

Keywords: fetal abnormalities, dog, kidney, gestational ultrasound, prenatal care. 


\section{INTRODUCTION}

The diagnosis and ultrasound evaluation of the fetal urinary tract as part of prenatal care in humans has been carried out for many years [9]. Still, by detecting possible abnormalities in the fetuses, the early treatment of urinary tract obstructions and infections can be done, preventing more serious damage to the individuals such as loss of kidney function [8]. In veterinary medicine, some studies indicate the possibility and importance of prenatal ultrasound evaluation of the kidneys and the normal development in dogs [3]. However, to date, no reports have been found that specifically describe ultrasound abnormalities of fetal kidneys in animals.

Among the main injuries that can compromise renal functionality, interstitial nephritis is an abnormality that causes decreased glomerular filtration, dilation of the renal pelvis and calyxes, resulting in progressive atrophy and increased renal parenchyma [14]. Acute interstitial nephritis is a frequent cause of acute kidney injury, and although it is reversible, the disease can progress, cause fibrosis and become chronic [10], leading to interstitial inflammation and tubular destruction [13].

The diagnosis is accomplished based in the history, blood and urine tests and the ultrasound examination, where, in adult, an increase in the echogenicity of the cortical and/or medullar region is seen, either in acute or chronic cases of interstitial nephritis [4]. Furthermore, a renal biopsy helps in the diagnosis and at staging of the disease [7].

This study aims to describe, during the prenatal examination, the B-mode and high-resolution ultrasound findings of the renal tissues of a canine fetus affected by interstitial nephritis.

\section{CASE}

A 4-year-old multiparous female Pug, approximately $5 \mathrm{~kg}$, was referred for ultrasonographic evaluation for pregnancy diagnosis, monitoring and evaluation of fetal viability and estimation of gestational age.

The gestational examination was performed using an ultrasound machine (ACUSON S2000) ${ }^{1}$ equipped with a $9.0 \mathrm{MHz}$ linear transducer and a high-resolution digital transducer with a frequency standardized at $17.0 \mathrm{MHz}$.
The first evaluation was carried out at 25 days of gestation, where four embryonic vesicles were detected with findings compatible with the gestational period and within normal limits. At 45 days, a new ultrasound evaluation was performed, and one of the fetuses was found to be underdeveloped or reduced in size when compared to the others (normal fetuses: biparietal diameter of $1.63 \mathrm{~cm}$ and abdominal diameter of $2.45 \mathrm{~cm}$; fetus with reduced sizes: biparietal diameter of $1.41 \mathrm{~cm}$ and abdominal diameter of $1.59 \mathrm{~cm}$ ). When using high definition ultrasonography (HD), both kidneys showed thickening of the cortical portion, associated with cortical hyperechogenicity and loss of corticomedullary ratio (1:1). In addition, the renal pelvis and ureters of the underdeveloped fetus were markedly dilated by an anechoic content (Figure 1) while the other fetuses did not show abnormalities, presenting normal development, compatible with the estimated gestational age.

At 58 days of gestation, the female was evaluated again through ultrasound, being possible to verify in the underdeveloped fetus that both kidneys still presented the abnormalities seen previously (Figure 2A). Additionally, a cystic structure in the cranial topography of the renal cortex was found in the right kidney (Figure 2B). Nonetheless, the other fetuses showed normal ultrasonographic findings, normal heartbeat, compatible with the ultrasound gestational age.

The delivery occurred naturally, without complications, however, one of the fetuses, the smallest one, was born dead while the others were healthy. The stillborn was sent to necropsy, where tissue from kidneys, brain, and liver were collected and fixed in a $10 \%$ buffered formaldehyde solution ( $\mathrm{pH}$ 7.4). The histological slides were prepared following the routine protocols, and hematoxylin-eosin staining ${ }^{2}$ was used to perform the histopathological analysis.

Upon the histopathological examination, only the renal tissue showed some abnormalities, characterized as chronic and discrete interstitial nephritis, with hemorrhagic areas and foci of glomerular sclerosis (Figure 3).

\section{DISCUSSION}

The importance of describing the occurrence of abnormalities diagnosed in prenatal care reinforces the need for a thorough examination of fetal development. Despite the bitch being considered healthy and showing no signs of complications during pregnancy, it 


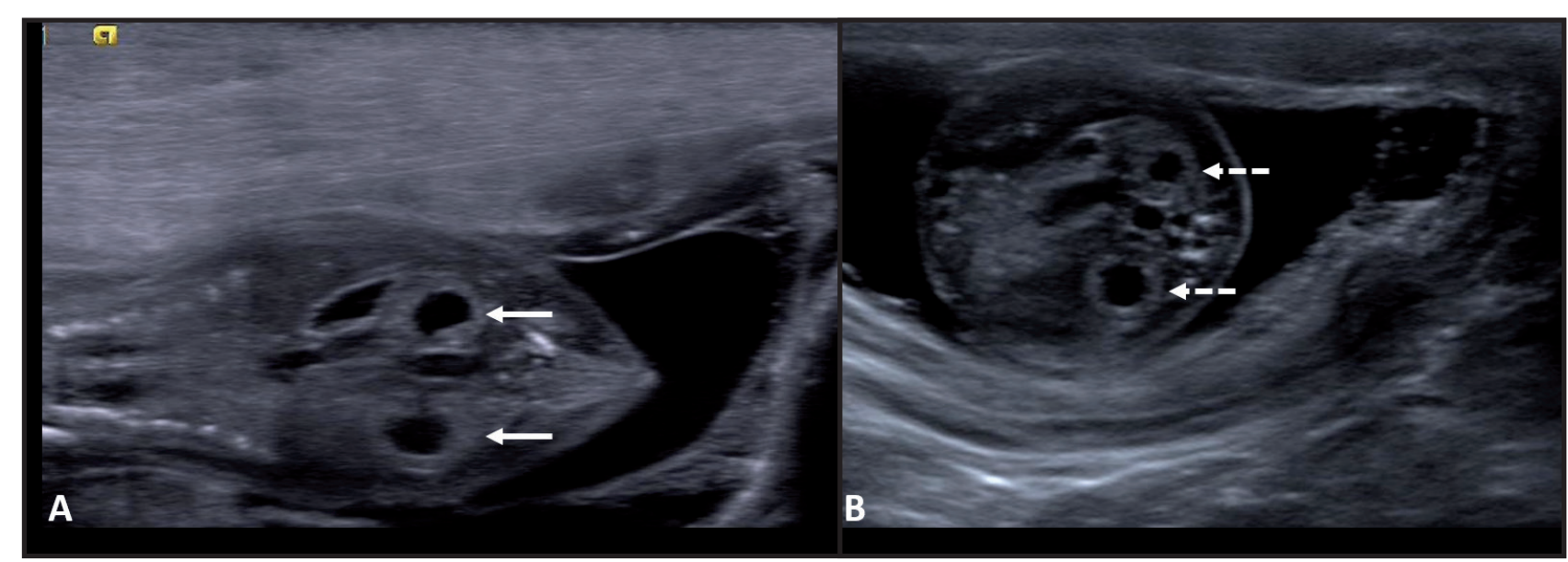

Figure 1. High-definition ultrasound image (HD) of a canine fetus at 45 days of gestation. A- Longitudinal section (continuous arrows). B- Cross-section of the kidneys (dotted arrows). Renal structures with thickened cortex and increased echogenicity are also noted, as well as the loss of the corticomedullary relationship, and renal pelvis markedly dilated by an anechoic content.

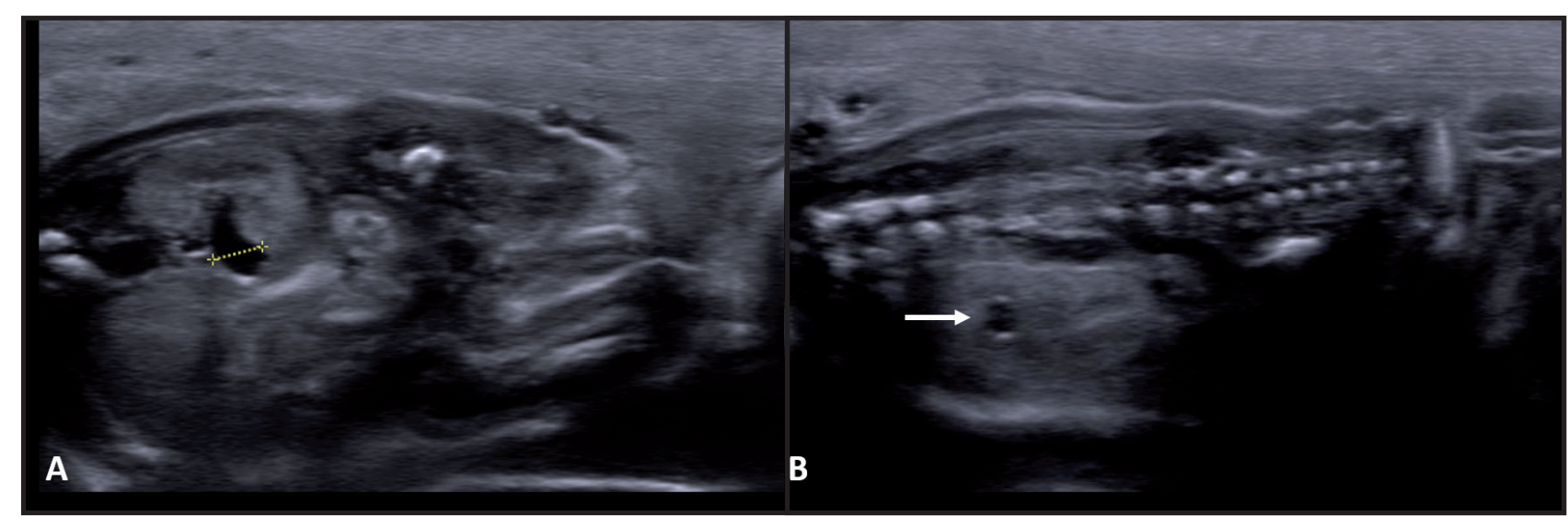

Figure 2. High definition ultrasound image (HD) of a canine fetus at 58 days of gestation. A- Left kidney showing enlarged ureter (between cursors). B- Right kidney showing cyst in the cortex (arrow).

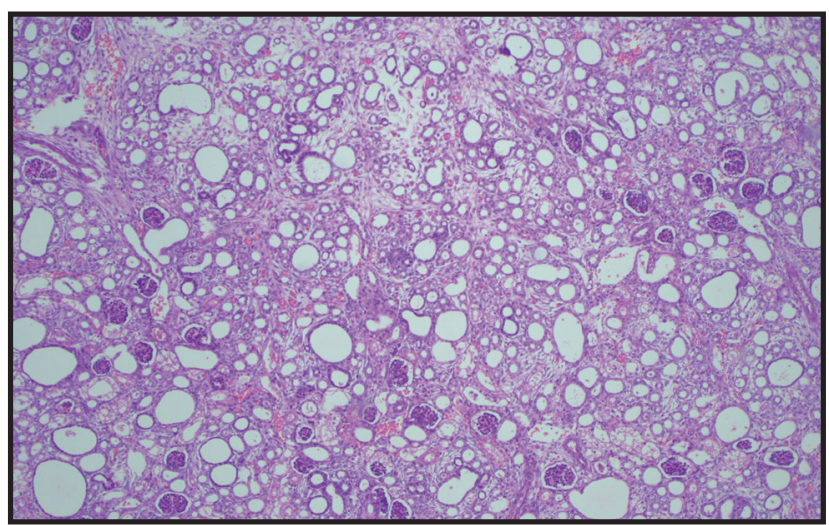

Figure 3. Histological image showing moderate multifocal glomerular sclerosis, interspersed with secondary hemorrhagic and necrotic foci organized in the interstitial region, permeated by aggregates of lymphocytes, plasmocytes, and macrophages. Diagnosis: Chronic interstitial nephritis.

was possible, through the ultrasound technique, to detect fetal underdevelopment and some anomalies, more specifically, in the kidneys. The successful and accurate prenatal diagnosis of urinary tract malformations can alter the course of pregnancy and treatment [8].

Generally, renal biopsy is necessary to assist in the diagnosis of abnormalities, however, this is an invasive procedure and is occasionally associated with complications [12]. On the other hand, the B and HD ultrasound techniques allow non-invasive and detailed morphological evaluations without the use of radiation $[7,16]$, which is an important characteristic, especially in pregnant patients.

Abnormalities related to the parenchyma and size of the renal pelvis in human fetuses have been used as a basis for detecting kidney diseases [1,2]. In this report, it was possible to verify that at 45 and 58 days of gestation, both kidneys of the underdeveloped fetus had hyperechogenic characteristics and cortical thickening. Differently, Gil et al. [5] reported that from 35 to 40 days of gestation the normal fetal kidney showed dilated renal pelvis, thickened cortex, and no cortical medullary definition. However, when assessing it at 55 days of gestation, the fetal kidney looks similar to an adult animal kidney, with a thin cortex, with corticomedullary definition, and without dilation of the renal pelvis. Thus, these findings raise a question about the possible establishment of abnormalities consistent with nephropathy. 
Chronic interstitial nephritis is described on histopathological examination as tubular interstitial nephritis associated with glomerular sclerosis and mild vascular changes [6], which corroborates the findings in the present study. Even though it is not the diagnosis for the chronic phase, histopathology is essential to exclude other diseases such as progressive glomerulonephritis and amyloidosis, in addition to specifying the degree of fibrosis, helping to define the prognosis.

Prenatal identification of renal anomalies offers broader options for decisions even during pregnancy, as they significantly affect perinatal morbidity and mortality [2], as observed in the present study, where the affected fetus was stillborn. Besides, prenatal diagnosis also works as an essential mechanism in the early detection of fetal abnormalities, which may present itself later in life, possibly with more advanced sequelae [2]. Therefore, the description of such deformities can help prevent disease, being this the first case reported in canine fetuses. Indeed, interstitial nephritis is mostly associated with the use of medicines [11], making the orientation to animal owners, regarding the indiscriminate use of drugs during the pregnancy of animals, essential. Still, it is important to mention that leptospirosis could be another cause of interstitial nephritis in domestic animals [14], and so, its prevention is essential.

The present study first described the ultrasonographic findings of the kidney of a canine fetus affected by chronic interstitial nephritis. The ultrasound examination was extremely important for the diagnosis of this disease in prenatal care. High-resolution ultrasound (HD) seems to provide a better assessment of fetal development, allowing the identification of detailed abnormalities in the kidney.

\section{MANUFACTURERS}

${ }^{1}$ Siemens AG. Munich, Bayern, Germany.

${ }^{2}$ Laborclin Produtos para Laboratórios Ltda. Pinhais, PR, Brazil.

Acknowledgments. The authors would like to thank the State of Sao Paulo Research Foundation (FAPESP protocol numbers: 2017/14957-6 and 2019/15282-8) and the National Council for Scientific and Technological Development for the research grant, productivity scholarship award (processes 430023/20189 and 309199/2017-4) and doctorate scholarship.

Declaration of interest. The authors report no conflicts of interest. The authors alone are responsible for the content and writing of the paper.

\section{REFERENCES}

1 Devriendt A., Cassart M., Massez A., Donner C. \& Avni F.E. 2013. Fetal kidneys: additional sonographic criteria of normal development. Prenatal Diagnosis. 33(13): 1248-1252. DOI: 10.1002/pd.4240

2 Dias T., Sairam S. \& Kumarasiri S. 2014. Ultrasound diagnosis of fetal renal abnormalities. Best Practice \& Research Clinical Obstetrics \& Gynaecology. 28(3): 403-415. DOI: 10.1016/j.bpobgyn.2014.01.009

3 Feliciano M.A.R., Cardilli D.J., Nepomuceno A.C., Crivalero R.M., Silva M.A.M., Coutinho L.N., Oliveira M.E.F. \& Vicente W.R.R. 2014. Echobiometrics kidney and renal artery triplex doppler of canine fetuses. Arquivo Brasileiro de Medicina Veterinária e Zootecnia. 66(2): 445-449. DOI: 10.1590/1678-41626120

4 Garcia P.H.S. \& Vac M.H. 2019. Rins e Ureters. In: Feliciano M.A.R., Assis A.R. \& Vicente W.R.R. (Eds). Ultrassonografia em Cães e Gatos. São Paulo: MedVet, pp.177-216.

5 Gil E.M.U., Garcia D.A.A., Giannico A.T. \& Froes T.R. 2018. Early results on canine fetal kidney development: Ultrasonographic evaluation and value in prediction of delivery time. Theriogenology. 107: 180-187. DOI: 10.1016/j. theriogenology.2017.11.015

6 Jayasumana C. 2019. Chronic Interstitial Nephritis in Agricultural Communities (CINAC) in Sri Lanka. Seminars in Nephrology. 39(3): 278-283. DOI: 10.1016/j.semnephrol.2019.02.006

7 Jayasumana C., Orantes C., Herrera R., Almaguer M., Lopez L., Silva L.C., Ordunez P., Siribaddana S., Gunatilake S. \& De Broe M.E. 2016. Chronic interstitial nephritis in agricultural communities: a worldwide epidemic with social, occupational and environmental determinants. Nephrology Dialysis Transplantation. 32(2): 234-241. DOI: 10.1093/ndt/gfw346

8 Kurjak A., Latin V., Mandruzzato G., D’Addario V. \& Rajhvajn B. 1984. Ultrasound diagnosis and perinatal management of fetal genito-urinary abnormalities. Journal of Perinatal Medicine. 12(6): 291-312. DOI: 10.1515/ jpme.1984.12.6.291

9 Lawson T.L., Foley W.D., Berland L.L. \& Clark K.E. 1981. Ultrasonic evaluation of fetal kidneys. Radiology. 138(1): 153-156. DOI: 10.1148/radiology.138.1.7455076 
10 Oliva-Damaso N., Oliva-Damaso E. \& Payan J. 2018. Acute and Chronic Tubulointerstitial Nephritis of Rheumatic Causes. Rheumatic Disease Clinics. 44(4): 619-633. DOI: 10.1016/j.rdc.2018.06.009

11 Praga M. \& González E. 2010. Acute interstitial nephritis. Kidney International. 77(11): 956-961. DOI: 10.1038/ ki.2010.89

12 Rivers B.J., Walter P.A., Polzin D.J. \& King V.L. 1997. Duplex doppler estimation of intrarenal pourcelot resistive index in dogs and cats with renal disease. Journal of Veterinary International Medicine. 11(4): 250-260, DOI: 10.1111/j.1939-1676.1997.tb00099.x.

13 Robertson L.J. 1986. Spontaneous renal disease in Dogs. Toxicologic Pathology. 14(1): 101-108. DOI: 10.1177/019262338601400112

14 Yang C.W. 2018. Leptospirosis Renal Disease: Emerging Culprit of Chronic Kidney Disease Unknown Etiology. Nephron. 138(2): 129-136. DOI:10.1159/000480691 